\title{
铜催化苯乙烯不对称嗍胺化反应
}

\author{
张涌灵 ${ }^{a, b}$ 王敏 $^{a} \quad$ 曹鹏 $^{a}$ 廖建*, $, a, b$ \\ ( ${ }^{a}$ 中国科学院成都生物研究所 天然产物研究中心 成都 610041) \\ ${ }^{b}$ 中国科学院大学 北京 100049)
}

\begin{abstract}
摘要 报道了一种以手性亚砜膦配体/铜络合物为催化剂的苯乙烯不对称硼胺化反应, 该方法以联嗍酸频哪醇酯 (pinB-Bpin)和亲电性胺试剂分别作为嗍源和氮源, 合成手性 $\beta$-氨基烷基嗍酯, 该产物可方便地转化为有用的手性 $\beta$-羟 胺类化合物。
\end{abstract}

关键词＼cjkstart铜催化; 手性亚砜膦配体; 苯乙烯; 不对称硼胺化; 手性 $\beta$-羊胺

\section{Copper-Catalyzed Enantioselective Aminoboration of Styrenes with Chiral Sulfoxide Phosphine Ligand

\author{
Zhang, Yongling ${ }^{a, b} \quad{\text { Wang, } \text { Min }^{a} \quad \text { Cao, Peng }}^{a} \quad$ Liao, Jian*, ${ }^{*, b}$ \\ $\left({ }^{a}\right.$ Natural Products Research Center, Chengdu Institute of Biology, Chinese Academy of Sciences, Chengdu 610041) \\ ( ${ }^{b}$ University of Chinese Academy of Sciences, Beijing 100049)
}

\begin{abstract}
To date, copper catalysis has become an attractive approach to access multifunctional alkylborons through borylative coupling processes, many important protocols such as carboboration, stannylboration and aminoboration were developed. Among these methods, however, there is no report involving enantioselective aminoboration of simple styrene substrates, which can generate a class of useful chiral compounds. In this work, an enantioselective $\mathrm{Cu}$-catalyzed aminoboration of styrenes by using a chiral sulfoxide-phosphine (SOP) ligand was developed, chiral $\beta$-aminoalkylboranes were obtained in satisfied yields and ee values, and these products can be readily converted to a class of valuable $\beta$-hydroxylalkylamines. A general procedure for the aminoboration of styrenes is as following: in glove box, $\mathrm{CuCl}(0.02 \mathrm{mmol})$, chiral sulfoxide phosphine $\mathbf{L 1}$ $(0.022 \mathrm{mmol})$ and $2.0 \mathrm{~mL}$ of dried tetrahydrofuran were added into a flame-dried tube, the resolved solution was stirred for $30 \mathrm{~min}$ at room temperature, then bis(pinacolato)diboron $\left(\mathrm{B}_{2} \mathrm{pin}_{2}\right)(0.3 \mathrm{mmol}), t$-BuOLi $(0.6 \mathrm{mmol})$ and styrene $(0.2 \mathrm{mmol})$ were added. The tube was taken out of the glove box and cooled to $0{ }^{\circ} \mathrm{C}$. Electrophilic amination reagent, $O$-benzoyl- $N, N$ dibenzylhydroxylamine $(\mathbf{2 a}, 0.3 \mathrm{mmol})$, was dissolved in $1.0 \mathrm{~mL}$ of ethyl acetate and added to the mixture, the resolved mixture was stirred at $0{ }^{\circ} \mathrm{C}$ for $24 \mathrm{~h}$. The crude product was filtered through a celite pad, concentrated and oxidized by $\mathrm{NaBO}_{3} \bullet 4 \mathrm{H}_{2} \mathrm{O}$. The mixture was extracted three times with ethyl acetate, concentrated and purified with silica gel chromatography to give the desired $\beta$-hydroxylalkylamines, the enantioselective excess of products were determined by chiral HPLC analysis. Broad substrate scope which related to steric and electronic effect were compatible in this catalysis under the standard conditions. To demonstrate the utility of this method, a gram scale experiment was performed and the desired product was obtained in $92 \%$ isolated yield and $90 \%$ ee. The benzyl group of products can be readily removed via a Pd/C-catalyzed hydrogenation process and the corresponding product with a free amino group in excellent yield (95\%).
\end{abstract}

Keywords Cu-catalyzed; chiral sulfoxide phosphine; styrenes; enantioselective aminoboration; $\beta$-hydroxylalkylamines

\section{1 引言}

有机嗍化合物是一类非常重要的有机合成砌块, 广 泛被应用于各类碳一碳键 $(\mathrm{C}-\mathrm{C})$ 和碳一杂键 $(\mathrm{C}-\mathrm{X})$ 形 成反应中 ${ }^{[1]}$, 因此, 对于这类化合物的合成方法学研究 一直是有机化学领域的热点之一. 过渡金属 $\left(\mathrm{Pd} / \mathrm{Pt}^{[2]}\right.$, $\mathrm{Cu}^{[3]}, \mathrm{Co} / \mathrm{Fe}^{[4]}, \mathrm{Ni}^{[5]}$ 催化的对碳碳不饱和键的硼化加成 反应能够方便、有效地构建烷基硼或烯基硼化合物，其 中, 铜催化因其低毒及相对廉价的优点得到了较为广泛
的关注，通过铜催化嗍化偶联反应 ${ }^{[6]}$ (嗍碳化，嗍锡化和 嗍胺化等)可以很好地构建多官能化烷基嗍化合物，在 有机合成中具有广泛的应用前景. $\alpha$-氨基硼酯类化合物 被广泛应用在药物、天然产物和活性分子的合成中 ${ }^{[7]}$. 铜催化的烯烃 1,2 -硼胺化反应 ${ }^{[8]}$ 是构建该类骨架较为理 想的方法之一. 2013 年, Miura 课题组 ${ }^{[8 a}$ 报道了首例以联 硼酸频哪醇酯(pinB-Bpin)和 $O$-苯甲酰基- $N, N$-二苄基羟 胺试剂 $\left(\mathrm{Bn}_{2} \mathrm{NOBz}\right)$ 分别作为亲核硼源和亲电氮源, 铜催 化实现了苯乙烯类化合物的嗍胺化反应. 同时对不对称

*E-mail: jliao@cib.ac.cn

Received April 12, 2017; published May 9, 2017.

Supporting information for this article is available free of charge via the Internet at http://sioc-journal.cn.

Project supported by the National Natural Science Foundation of China (Nos. 21472184, 21572218 and 21402186).

项目受国家自然科学基金(Nos. 21472184, 21572218 和 21402186)资助. 
嗍胺化进行了初步尝试, 以 $(S, S)$-Me-Duphos 作为手性 配体, 实现了反式- $\beta$-甲基苯乙烯的硼胺化, 得到较好的 对映选择性( $80 \%$ ～ $86 \%$ ee) 和中等到较好的收率(51\% $83 \%$ ). 随后, Miura 和 Shi 课题组分别报道了系列特定结 构烯烃如双环烯烃 ${ }^{[8 \mathrm{e}]}$ 、烯基硅烷 ${ }^{[8]}$ 、烯基碀酯 ${ }^{[8 \mathrm{~g}]}$ 和含环 丙烷烯烃 ${ }^{[8 b, 8 h]}$ 等的不对称硼胺化反应. 到目前为止, 针 对大量商业可得的简单苯乙烯的不对称嗍胺化反应尚 没有报道. 我们课题组近期的研究兴趣集中在通过我们 自己设计的手性亚砜配体去实现简单烯烃的不对称官 能化, 特别是不对称双官能化. 最近, 我们实现了手性 亚砜膦配体(SOP)/铜络合物催化苯乙烯的嗍碳化反应和 嗍锡化反应, 获得了优秀的收率和对映选择性 ${ }^{[9]}$. 在这 些前期研究工作基础上, 我们在本工作中对铜催化苯乙 烯的不对称嗍胺化反应进行了研究, 以手性亚砜膦配体 (SOP)/铜络合物作为催化剂, 实现了简单苯乙烯的不对 称 1,2-嗍胺化反应, 获得了较高的收率和最高达 95\%的 对映选择性. 该方法为手性氨基硼化合物提供了一条高 效的合成路径.

\section{2 结果与讨论}

我们选取苯乙烯(1a)、亲电性胺试剂(2a)和联硼酸 频哪醇酯(pinB-Bpin)作为反应底物, 用手性亚砜膦配体 (SOP) $\mathbf{L 1}$ 和 $\mathrm{CuCl}$ 原位络合生成活性催化剂, 以叔丁醇 锂 $(\mathrm{LiOBu}-t)$ 为碱, 四氢呋喃(THF)为溶剂, 设定反应温 度为 $0{ }^{\circ} \mathrm{C}$, 对反应进行了初步尝试(表 1, Entry 1). 反应 $24 \mathrm{~h}$ 后, 以较高的收率(85\%)和很好的对映选择性(89\% $e e)$ 得到烯烃的嗍胺化产物. 但由于硼胺化产物在硅胶 柱层析分离纯化过程中不稳定, 容易发生分解, 因此在 实际后处理过程中, 我们将得到的硼胺化产物直接氧化 为稳定的 $\beta$-羟胺化合物 3a, 并对其进行表征分析. 通过 测定 $3 \mathbf{a}$ 的比旋光值, 与已知文献进行比对 ${ }^{[10]}$, 确定了 产物 $3 \mathbf{a}$ 的绝对构型为 $S$. 为了进一步提高反应的对映选 择性, 我们对反应条件进行了优化.

我们首先考察了手性配体(表 1, Entries 2～5), 包括 其它我们研究小组所发展的 SOP 配体(详细信息见 SI) 和几种商业性双膦配体. 与手性亚砜膦配体 L1 相比, $(S, S)$-Me-Duphos (L2), $(R)$-BINAP $(\mathbf{L 3}),(S, S)$-BDPP (L4) 以及 $(R)-\mathrm{SEGPHOS}(\mathbf{L 5})$ 不管是在反应活性还是对映选 择性上, 都没有表现出更好的结果(表 1, Entries 2 5). 随后, 我们考察了反应溶剂对反应的影响(表 1, Entries 6 12). 以乙腈作为溶剂, 反应的对映选择性基本相当, 但是活性却明显降低(表 1, Entry 6); 甲基叔丁基醚 (MTBE)给出的收率和对映选择性均较低(表 1, Entry 7); 该反应在甲苯中无法得到目标产物(表 1, Entry 8); 当使 用乙酸乙酯(EA)作为溶剂时, 反应的对映选择性得到明 显提高 $(94 \% e e)$, 但是收率较低, 这主要是由亲电性胺 试剂 $2 \mathbf{a}$ 在乙酸乙酯中溶解性较差所致(表 1, Entry 9). 值
得高兴的是，我们综合考虑了四氢呋喃的高收率和乙酸 乙酯的高对映选择性, 选取了混合溶剂(THF/EA, $V: V$ $=2 / 1$ ), 可同时实现产物 3a 的高收率和高对映选择性 (表 1, Entry 12). 最后我们考察了碱对反应的影响(表 1 , Entries 13 15). 由于亲电性胺试剂 $\mathbf{2 a}$ 的转酯化作用, 叔丁醇钾只能得到痕量的产物(表 1 , Entry 13); 氢氧化 钾和甲醇锂均可得到相当的对映选择性, 但是收率较低 (表 1, Entry 14, 15). 此外我们对亲电性胺试剂苯甲酰基 取代基以及氮上不同取代基也进行了系统考察，但并没 有获得更好的结果(见 SI).

表 1 反应条件优化 ${ }^{a}$

Table 1 Reaction conditions screening

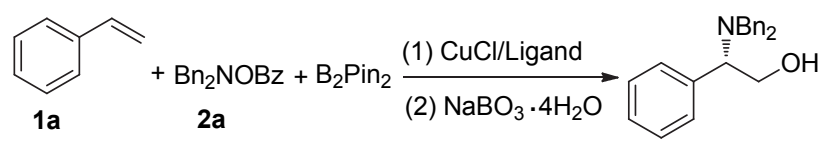

3a<smiles>CCOc1c(OC)ccc(S(=O)C(C)(C)C)c1P(=O)(O)c1ccccc1</smiles><smiles>[Z10]C1CCC(C)(C)P1c1ccccc1P1CCC[C@@H]1C</smiles><smiles>c1ccc(-c2ccc3ccccc3c2-c2c(P(c3ccccc3)c3ccccc3)ccc3ccccc23)cc1</smiles>

L3

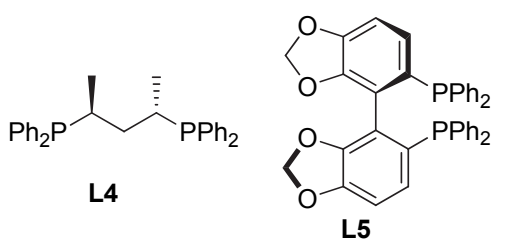

\begin{tabular}{cccccc}
\hline Entry & $\mathbf{L}$ & Base & Solvent $(V: V)$ & Yield $^{b} / \%$ & $e e^{c} / \%$ \\
\hline 1 & $\mathbf{L 1}$ & $t$-BuOLi & THF & 85 & 89 \\
2 & $\mathbf{L 2}$ & $t$-BuOLi & THF & 79 & -30 \\
3 & $\mathbf{L 3}$ & $t$-BuOLi & THF & 60 & 50 \\
4 & $\mathbf{L 4}$ & $t$-BuOLi & THF & 64 & 50 \\
5 & $\mathbf{L 5}$ & $t$-BuOLi & THF & 49 & 67 \\
6 & $\mathbf{L 1}$ & $t$-BuOLi & $\mathrm{CH}_{3} \mathrm{CN}$ & 48 & 88 \\
7 & $\mathbf{L 1}$ & $t$-BuOLi & MTBE & 57 & 82 \\
8 & $\mathbf{L 1}$ & $t$-BuOLi & Tol & trace & nd \\
$9^{d}$ & $\mathbf{L 1}$ & $t$-BuOLi & EA & 40 & 94 \\
$10^{d}$ & $\mathbf{L 1}$ & $t$-BuOLi & THF/EA $(1: 2)$ & 50 & 94 \\
$11^{d}$ & $\mathbf{L 1}$ & $t$-BuOLi & THF/EA $(1: 1)$ & 56 & 92 \\
$12^{d}$ & $\mathbf{L 1}$ & $t$-BuOLi & THF/EA $(2: 1)$ & $83(76)^{e}$ & 93 \\
$13^{d}$ & $\mathbf{L 1}$ & $t$-BuOK & THF/EA $(2: 1)$ & trace & $\mathrm{nd}^{f}$ \\
$14^{d}$ & $\mathbf{L 1}$ & $\mathrm{KOH}$ & $\mathrm{THF} / \mathrm{EA}(2: 1)$ & 71 & 95.5 \\
$15^{d}$ & $\mathbf{L 1}$ & $\mathrm{LiOMe}$ & $\mathrm{THF} / \mathrm{EA}(2: 1)$ & 56 & 96 \\
\hline
\end{tabular}

${ }^{a}$ Conducted with 1a $(0.2 \mathrm{mmol}), \mathrm{B}_{2}(\text { pin })_{2}(0.3 \mathrm{mmol}), \mathrm{Bn}_{2} \mathrm{NOBz}(0.3 \mathrm{mmol})$, $\mathrm{CuCl}(10 \mathrm{~mol} \%)$, ligand $(11 \mathrm{~mol} \%)$, base $(0.6 \mathrm{mmol})$ in solvent $(2.0 \mathrm{~mL})$ at $0{ }^{\circ} \mathrm{C}$ for $24 \mathrm{~h}$. Then, the resulting mixture was filtered through celite, concentrated, and oxidized by $\mathrm{NaBO}_{3} \cdot 4 \mathrm{H}_{2} \mathrm{O}$ (8 equiv.) in THF/ $\mathrm{H}_{2} \mathrm{O}(V: V=1: 1,4$ $\mathrm{mL})$ for $5 \mathrm{~h}$ at $20{ }^{\circ} \mathrm{C} .{ }^{b}$ Determined by ${ }^{1} \mathrm{H}$ NMR spectroscopy. ${ }^{c}$ Determined by chiral HPLC. ${ }^{d}$ Solvent is $3.0 \mathrm{~mL} .{ }^{e}$ Value in parentheses is isolated yield of $3 \mathbf{a}$. ${ }^{f}$ nd $=$ not detected. 
综合考虑上述实验结果, 我们确定了反应的最优条 件: 以 $10 \mathrm{~mol} \% \mathrm{CuCl}$ 为催化剂前体, $11 \mathrm{~mol} \% \mathrm{L1}$ 为手性 配体, 以四氢呋喃/乙酸乙酯 $(V: V=2: 1)$ 为溶剂, 叔丁 醇锂作为碱, $0{ }^{\circ} \mathrm{C}$ 条件下反应 $24 \mathrm{~h}$.

在最优条件下, 我们对铜催化苯乙烯不对称硼胺化 反应底物普适性进行了考察(表 2). 针对苯环上取代基 电子效应和位阻效应, 各种取代苯乙烯底物被引入到反 应体系中, 均可以很好地与亲电性胺试剂 $2 \mathbf{a}$ 和喼试剂 $\left(\mathrm{B}_{2} \mathrm{pin}_{2}\right)$ 发生反应，目标产物以较好的收率和对映选择 性获得(产率 55\% 78\%, 60\% 95\% ee). 卤素取代 $(\mathrm{Cl}$, $\mathrm{Br}, \mathrm{F})$ 苯乙烯在该反应中耐受, 可取得中等结果. 当苯 环取代基为供电子基团时, 相对于缺电子烯烃, 可获得 较高的对映选择性. 对位强吸电子基取代苯乙烯(3q)因 发生强烈的质子化(即硼氢化)竞争副反应, 导致较低的 收率和对映选择性. 比较有趣的是, 缺电子烯烃和富电 子烯烃在位阻方面表现出了不一样的趋势. 例如, 间位 (3f)和对位 (3k) 甲基取代的富电子苯乙烯可以获得比邻 位(3b)甲基苯乙烯更好的对映选择性. 然而卤素 $(\mathrm{Cl}, \mathrm{Br})$ 取代的缺电子苯乙烯却表现出了相反的结果: 邻位取代 $(3 c, 3 d)$ 的苯乙烯比间位 $(3 g, 3 h)$ 和对位 $(3 \mathrm{~m}, 3 \mathrm{n})$ 取代的
苯乙烯表现出了更好的对映选择性. 相同位置取代基的 位阻增大有利于对映选择性，但是反应活性会有一定程 度的降低. 当烯烃底物是 2-乙烯基噻吩时, 反应同样可 以得到很好的对映选择性，但是收率却很低(3u, 29\%, $89 \% \mathrm{ee}$ ), 可能的原因是底物中硫原子与铜有配位作用 导致催化剂的活性降低. 此外，非芳香性基团取代烯烃 如降冰片二烯也能顺利得到预期产物, 有较好的收率和 对映选择性 $(3 \mathrm{v}, 75 \%, 80 \% \mathrm{ee})$, 其活性可能来源于其特 殊二烯结构和骨架具有较强的张力, 其它非芳香烯烃如 环己烯、脂肪链取代的端烯 $(3 \mathbf{w} \sim 3 y)$ 等在该反应体系中 则无活性. 由于位阻原因, $\beta$-取代苯乙烯 $(3 z)$ 在该体系同 样无反应活性, 本工作研究结果表明, 亚砜膦配体与双 膦配体如 DuPhos 在底物适用范围上具有互补性 ${ }^{[8 \mathrm{a}]}$.

为了体现该反应的实用性, 我们对放大实验进行了 尝试. 在标准反应条件下, 将模板反应规模扩大到克级 (1a, $1.04 \mathrm{~g})$, 获得了比小规模反应更好分离收率( $92 \% \mathrm{vs}$ $76 \%$ )和基本保持的对映选择性(90\% ee). 同时，在温和 的反应条件下, 通过钯/碳催化氢化, 可高效地脱除产物 的氮茮基保护基(95\%), 得到 $(S)-(+)$-2-苯甘氨醇.

表 2 硼胺化底物范围考察 ${ }^{a}$

Table 2 Substrate scope of aminoboration

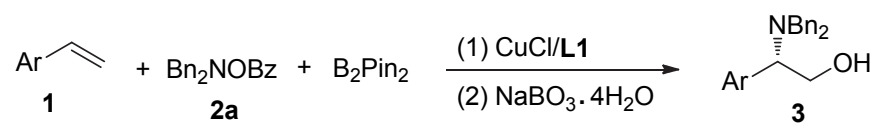

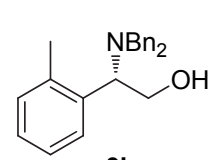

3b

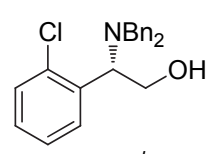

$3 \mathbf{c}^{d}$

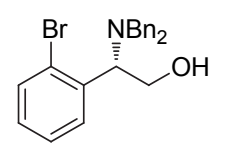

$3 \mathbf{d}^{e}$

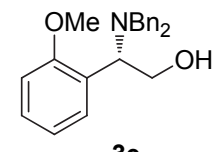
$3 \mathbf{e}$
$67 \%, 87 \%$ ee

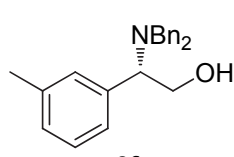

$3 \mathbf{f}$ $63 \%, 90 \%$ ee<smiles>CCCC[C@H](CO)c1cccc(Cl)c1</smiles>

$3 \mathbf{g}$ $58 \%, 88 \%$ ee $58 \%, 62 \%$ ee<smiles>O=C(C[C@H](CO)c1cccc(Br)c1)c1ccccc1</smiles>

$62 \%, 68 \%$ ee

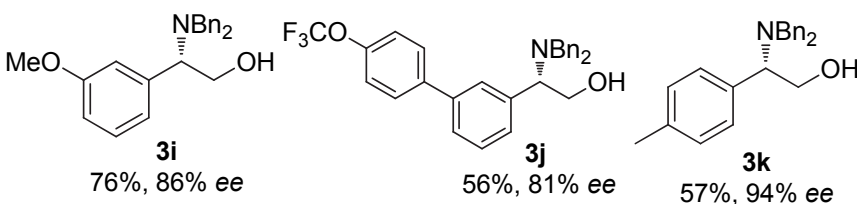

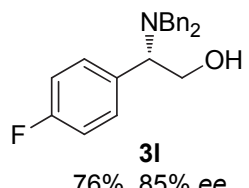<smiles>OC[C@H](c1ccccc1)c1ccc(Cl)cc1</smiles><smiles>OC[C@@H](Cc1ccccc1)c1ccc(Br)cc1</smiles>
$69 \%, 67 \%$ ee $57 \%, 77 \%$ ee<smiles>CC(C)(C)[NH2+]C(CO)c1ccc(C(C)(C)C)cc1</smiles>
$43 \%, 94 \%$ ee<smiles></smiles><smiles>CCCCC[C@H](CO)c1ccc(C(F)(F)F)cc1</smiles>
$57 \%, 60 \%$ ee<smiles>COc1ccc(C(CO)C(=O)c2ccccc2)cc1</smiles>

$78 \%, 94 \%$ ee<smiles>OCC([18OH])c1ccc2ccccc2c1</smiles>

$56 \%, 76 \%$ ee<smiles>COc1ccc(C([Pb])CO)cc1OC</smiles>

$62 \%, 95 \%$ ee<smiles>[15NH2]C(CO)c1cccs1</smiles>

$3 \mathbf{u}$

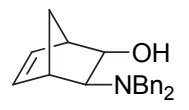

3v

$29 \%, 89 \%$ ee

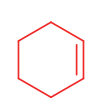

$1 w$

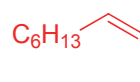

$1 \mathrm{x}$

no reaction

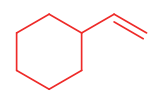

$1 y$ no reaction

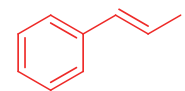

$1 z$

${ }^{a}$ Reaction conditions unless otherwise noted: 1a $(0.2 \mathrm{mmol}), \mathrm{B}_{2}(\mathrm{pin}){ }_{2}(0.3 \mathrm{mmol}), \mathbf{2 a}(0.3 \mathrm{mmol}), \mathrm{CuCl}(10 \mathrm{~mol} \%), \mathbf{L 1}(11 \mathrm{~mol} \%), t$-BuOLi $(0.6 \mathrm{mmol})$ in $3.0 \mathrm{~mL}$ mixed solvent (THF/EA, $V: V=2: 1)$ at $0{ }^{\circ} \mathrm{C}$ for $24 \mathrm{~h}$. Then, the resulting mixture was filtered through celite, concentrated, and oxidized by $\mathrm{NaBO}_{3} \bullet 4 \mathrm{H}_{2} \mathrm{O}(8$ equiv.) in THF/ $\mathrm{H}_{2} \mathrm{O}(V: V=1: 1,4 \mathrm{~mL})$ for $5 \mathrm{~h}$ at $20{ }^{\circ} \mathrm{C} .{ }^{b}$ Isolated yield. ${ }^{c}$ Determined by chiral HPLC. ${ }^{d}$ Conducted at room temperature for $24 \mathrm{~h} .{ }^{e} \mathrm{Conducted}$ with $\mathrm{B}_{2}$ (pin) $)_{2}(0.6 \mathrm{mmol}), 2 \mathrm{a}(0.6 \mathrm{mmol})$ at $0{ }^{\circ} \mathrm{C}$ for $24 \mathrm{~h}$ 


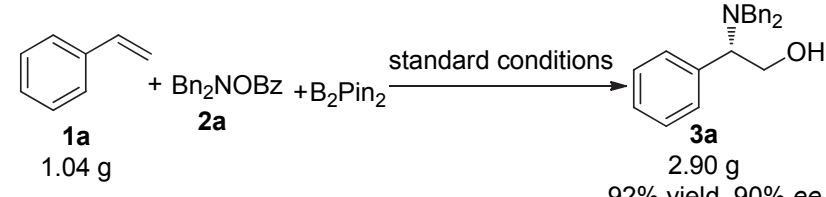

$92 \%$ yield, $90 \%$ ee
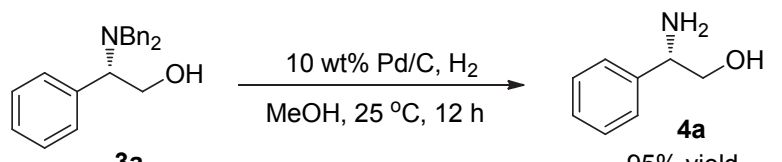

$95 \%$ yield

Reaction conditions: $10 \mathrm{wt} \% \mathrm{Pd} / \mathrm{C}$ wetted with ca. $55 \%$ water $(0.04$ $\mathrm{mmol}),(S)-3 a(0.4 \mathrm{mmol})$ in $\mathrm{MeOH}(3.0 \mathrm{~mL})$ under hydrogen by balloon. The suspension was stirred at $25{ }^{\circ} \mathrm{C}$ for $12 \mathrm{~h}$.

图式 1 放大实验和 $3 \mathbf{a}$ 脱保护反应

Scheme 1 Scaled-up experiment and deprotection of 3a

\section{3 结论}

我们以铜/手性亚砜膦络合物作为催化剂，通过对 反应条件的优化, 实现了简单苯乙烯底物的不对称 $1,2-$ 嗍胺化反应, 取得了较高的收率和最高达 $95 \%$ 的对映选 择性. 通过温和的氧化, 可将产物的嗍基团转化为羟基, 为手性 $\beta$-羊弪胺类化合物的合成提供了一种有效的方法.

\section{References}

[1] (a) Pelter, A.; Smith, K.; Brown, H. C. Borane Reagents, Academic Press, London, 1988; (b) Miyaura, N.; Suzuki, A. Chem. Rev. 1995, 95, 2457; (c) Davison, M.; Hughes, A. K.; Marder, T. B.; Wade, K. Contemporary Boron Chemistry, RSC, Cambridge, U. K., 2000; (d) Boronic Acids, 2nd ed.; Ed.: Hall, D. G., Wiley-VCH, Weinheim, Germany, 2011.

[2] (a) Ishiyama, T.; Matsuda, N.; Miyaura, N.; Suzuki, A. J. Am. Chem. Soc. 1993, 115, 11018; (b) Ishiyama, T.; Matsuda, N.; Murata, M.; Ozawa, F.; Suzuki, A.; Miyaura, N. Organometallics 1996, 15, 713; (c) Lesley, G.; Nguyen, P.; Taylor, N. J.; Marder, T. B. Organometallics 1996, 15, 5137; (d) Ishiyama, T.; Yamamoto, M.; Miyaura, N. Chem. Commun. 1996, 2073; (e) Ishiyama, T.; Yamamoto, M.; Miyaura, N. Chem. Commun. 1997, 689; (f) Thomas, R. L.; Souza, F. E. S.; Marder, T. B. J. Chem. Soc., Dalton Trans. 2001, 1650; (g) Yang, F.-Y.; Cheng, C.-H. J. Am. Chem. Soc. 2001, 123, 761; (h) Burks, H. E.; Kliman, L. T.; Morken, J. P. J. Am. Chem. Soc. 2009, 131, 9134; (i) Kliman, L. T.; Mlynarski, S. N.; Morken, J. P. J. Am. Chem. Soc. 2009, 131, 13210; (j) Iwadate, N.; Suginome, M. J. Am. Chem. Soc. 2010, 132, 2548; (k) Coombs, J. R.; Haeffner, F.; Kliman, L. T.; Morken, J. P. J. Am. Chem. Soc. 2013, 135, 11222; (1) Coombs, J. R.; Zhang, L.; Morken, J. P. J. Am. Chem. Soc. 2014, 136, 16140; For review, see: Ishiyama, T.; Ishida, K.; Miyaura, N. Tetrahedron 2001, 57, 9813 and references therein.

[3] Selected examples, see: (a) Ito, H.; Yamanaka, H.; Tateiwa, J.-i.; Hosomi, A. Tetrahedron Lett. 2000, 41, 6821; (b) Zhu, W.; Ma, D. Org. Lett. 2006, 8, 261; (c) Beenen, M. A.; An, C.; Ellman, J. A. J. Am. Chem. Soc. 2008, 130, 6910; (d) Lee, J. E.; Yun, J. Angew. Chem., Int. Ed. 2008, 47, 145; (e) Lipshutz, B. H.; Boskovic, Z. V.; Aue, D. H. Angew. Chem., Int. Ed. 2008, 47, 10183; (f) Chen, I. H.; Yin, L.; Itano, W.; Kanai, M.; Shibasaki, M. J. Am. Chem. Soc. 2009, 131, 11664; (g) Kleeberg, C.; Dang, L.; Lin, Z.; Marder, T. B. Angew. Chem., Int. Ed. 2009, 48, 5350; (h) Lillo, V.; Prieto, A.; Bonet, A.; Díaz-Requejo, M. M.; Ramírez, J. s.; Pérez, P. J.; Fernández, E. Organometallics 2009, 28, 659; (i) Noh, D.; Chea, H.; Ju, J.; Yun, J.
Angew. Chem., Int. Ed. 2009, 48, 6062; (j) O'Brien, J. M.; Lee, K. S.; Hoveyda, A. H. J. Am. Chem. Soc. 2010, 132, 10630; (k) Lee, J C.; McDonald, R.; Hall, D. G. Nat. Chem. 2011, 3, 894; (1) Moure, A. L.; Arrayas, R. G.; Carretero, J. C. Chem. Commun. 2011, 47, 6701; (m) Solé, C.; Whiting, A.; Gulyás, H.; Fernández, E. Adv. Synth. Catal. 2011, 353, 376; (n) Burns, A. R.; Solana Gonzalez, J.; Lam, H. W. Angew. Chem., Int. Ed. 2012, 51, 10827; (o) Ito, H.; Kubota, K. Org. Lett. 2012, 14, 890; (p) Yang, C. T.; Zhang, Z. Q.; Tajuddin, H.; Wu, C. C.; Liang, J.; Liu, J. H.; Fu, Y.; Czyzewska, M.; Steel, P. G.; Marder, T. B.; Liu, L. Angew. Chem., Int. Ed.. 2012, 51, 528; (q) Feng, X.; Jeon, H.; Yun, J. Angew. Chem., Int. Ed. 2013, 52, 3989; (r) Semba, K.; Nakao, Y. J. Am. Chem. Soc. 2014, 136 , 7567; (s) Smith, K. B.; Logan, K. M.; You, W.; Brown, M. K. Chem. Eur. J. 2014, 20, 12032; (t) Logan, K. M.; Smith, K. B.; Brown, M. K. Angew. Chem., Int. Ed. 2015, 54, 5228; (u) Su, W.; Gong, T. J.; Lu, X.; Xu, M. Y.; Yu, C. G.; Xu, Z. Y.; Yu, H. Z.; Xiao, B.; Fu, Y. Angew. Chem., Int. Ed. 2015, 54, 12957; (v) Liu, Q.; Tian, B.; Tian, P.; Tong, X.; Lin, G.-Q. Chin. J. Org. Chem. 2015, 35, 1; (刘强, 田 兵, 田平, 童晓峰, 林国强, 有机化学, 2015, 35, 1.) (w) Semba, K.; Ohtagaki, Y.; Nakao, Y. Org. Lett. 2016, 18, 3956; (x) Smith, J. J.; Best, D.; Lam, H. W. Chem. Commun. 2016, 52, 3770; (y) Logan, K. M.; Brown, M. K. Angew. Chem., Int. Ed. 2017, 56, 851.

[4] Selected examples, see: (a) Adams, C. J.; Baber, R. A.; Batsanov, A. S.; Bramham, G.; Charmant, J. P.; Haddow, M. F.; Howard, J. A.; Lam, W. H.; Lin, Z.; Marder, T. B.; Norman, N. C.; Orpen, A. G. Dalton Trans. 2006, 1370; (b) Obligacion, J. V.; Chirik, P. J. Org. Lett. 2013, 15, 2680; (c) Obligacion, J. V.; Chirik, P. J. J. Am. Chem. Soc. 2013, 135, 19107. (d) Zhang, L.; Peng, D.; Leng, X.; Huang, Z. Angew. Chem., Int. Ed. 2013, 52, 3676; (e) Cao, Y.; Zhang, Y.; Zhang, L.; Zhang, D.; Leng, X.; Huang, Z. Org. Chem. Front. 2014, 1, 1101; (f) Chen, J.; Xi, T.; Lu, Z. Org. Lett. 2014, 16, 6452; (g) Chen, J.; Xi, T.; Ren, X.; Cheng, B.; Guo, J.; Lu, Z. Org. Chem. Front. 2014, 1, 1306; (h) Zhang, L.; Zuo, Z.; Leng, X.; Huang, Z. Angew. Chem., Int. Ed. 2014, 53, 2696; (i) Zhang, L.; Zuo, Z.; Wan, X.; Huang, Z. J. Am. Chem. Soc. 2014, 136, 15501; (j) Guo, J.; Chen, J.; Lu, Z. Chem. Commun. 2015, 51, 5725; (k) Zhang, L.; Huang, Z. J. Am. Chem. Soc. 2015, 137, 15600; (1) Zhang, H.; Lu, Z. ACS Catal. 2016, 6, 6596; (m) Zuo, Z.; Huang, Z. Org. Chem. Front. 2016, 3, 434; (n) Zuo, Z.; Yang, J.; Huang, Z. Angew. Chem., Int. Ed. 2016, 55, 10839; (o) Xi, T.; Lu, Z. ACS Catal. 2017, 7, 1181 .

[5] (a) Suginome, M.; Matsuda, T.; Yoshimoto, T.; Ito, Y. Org. Lett. 1999, 1, 1567; (b) Suginome, M.; Shirakura, M.; Yamamoto, A. J. Am. Chem. Soc. 2006, 128, 14438; (c) Ely, R. J.; Morken, J. P. J. Am. Chem. Soc. 2010, 132, 2534.

[6] Reviews: (a) Semba, K.; Fujihara, T.; Terao, J.; Tsuji, Y. Tetrahedron 2015, 71, 2183. (b) Liu, Y.; Zhang, W. Chin. J. Org. Chem. 2016, 36, 2249. (刘媛媛, 张万斌, 有机化学, 2016, 36, 2249.)

[7] (a) Bloch, R. Chem. Rev. 1998, 98, 1407; (b) Ramadhar, T. R.; Batey, R. A. Synthesis 2011, 1321; (c) Yus, M.; González-Gómez, J. C.; Foubelo, F. Chem. Rev. 2013, 113, 5595 .

[8] (a) Matsuda, N.; Hirano, K.; Satoh, T.; Miura, M. J. Am. Chem. Soc. 2013, 135, 4934; (b) Sakae, R.; Matsuda, N.; Hirano, K.; Satoh, T.; Miura, M. Org. Lett. 2014, 16, 1228; (c) Parra, A.; Amenos, L.; Guisan-Ceinos, M.; Lopez, A.; Garcia Ruano, J. L.; Tortosa, M. J. Am. Chem. Soc. 2014, 136, 15833; (d) Sakae, R.; Hirano, K.; Miura, M. J. Am. Chem. Soc. 2015, 137, 6460; (e) Sakae, R.; Hirano, K.; Satoh, T.; Miura, M. Angew. Chem., Int. Ed. 2015, 54, 613; (f) Kato, K.; Hirano, K.; Miura, M. Angew. Chem., Int. Ed. 2016, 55, 14400; (g) Nishikawa, D.; Hirano, K.; Miura, M. Org. Lett. 2016, 18, 4856; (h) Shi, M. Chem. Commun. 2016, 52, 5273.

[9] (a) Chen, B.; Cao, P.; Yin, X.; Liao, Y.; Jiang, L.; Ye, J.; Wang, M.; Liao, J. ACS Catal. 2017, 7, 2425; (b) Jia, T.; Cao, P.; Wang, B.; Lou, Y.; Yin, X.; Wang, M.; Liao, J. J. Am. Chem. Soc. 2015, 137, 13760; (c) Jia, T.; Cao, P.; Wang, D.; Lou, Y.; Liao, J. Chem. Eur. J. 2015, 21, 4918.

[10] Metro, T. X.; Appenzeller, J.; Pardo, D. G.; Cossy, J. Org. Lett. 2006, 8,3509 . 